Proceedings of the 46th International School and Conference on the Physics of Semiconductors "Jaszowiec" 2017, Szczyrk

\title{
Microhardness and the Young Modulus of Thin, MBE-Grown, (Sn,Mn)Te Layers Containing up to $8 \%$ of Mn
}

\author{
S. AdAmiak ${ }^{a}$, M. ZięBA ${ }^{b}$, R. Minikayev ${ }^{b}$, A. Reszka $^{b}$, B. TAliashvili ${ }^{b}$ \\ AND W. SZUSZKIEWICZ ${ }^{a, b, *}$ \\ ${ }^{a}$ Faculty of Mathematics and Natural Sciences, University of Rzeszów, S. Pigonia 1, PL-35310 Rzeszów, Poland \\ ${ }^{b}$ Institute of Physics, Polish Academy of Sciences, Aleja Lotnikow 32/46, PL-02668 Warsaw, Poland
}

\begin{abstract}
The thin layers of ( $\mathrm{Sn}, \mathrm{Mn}) \mathrm{Te}$ solid solution were grown by molecular beam epitaxy onto (111)-oriented $\mathrm{BaF}_{2}$ substrates and characterized by scanning electron microscopy, atomic force microscopy, energy dispersive X-ray spectrometry, and X-ray diffraction methods. The epitaxial character of the growth was confirmed. All the layers exhibited a regular (fcc) structure of the rock-salt type and were (111)-oriented, their thickness was close to about $1 \mu \mathrm{m}$. The layers contained up to $8 \%$ of $\mathrm{Mn}$. The microhardness and the Young modulus values were determined by the nanoindentation measurements. The Berkovich type of the intender was applied, the maximum applied load was equal to $1 \mathrm{mN}$. The results of measurements demonstrated a lack of the composition dependence of the Young modulus value. A slight increase of the microhardness value with an increasing $\mathrm{Mn}$ content in the (Sn,Mn)Te solid solution was observed.
\end{abstract}

DOI: 10.12693/APhysPolA.132.347

PACS/topics: 62.20.de, 62.20.Qp, 68.60.Bs

\section{Introduction and motivation}

In the past few years, the lead and thin chalcogenides as well as various solid solutions containing these compounds have been attracting a lot of attention. Indeed, there have been multiple reasons for this. First of all, the lead telluride is one of the chalcogenides, which is considered as an extremely useful in the field of thermoelectricity and infrared detection. The lattice dynamics of $\mathrm{PbTe}$ and that of SnTe have recently been brought into an extensive debate because of some new physical phenomena that were observed in these compounds [1,2]. On the other hand, SnTe and selected solid solutions obtained on the basis of IV-VI compounds, like, e.g., (Pb,Sn)Se, in some composition range exhibits a presence of topological crystalline insulator states $[3,4]$. Despite a variety of experimental methods being applied to study the physical properties of all the IV-VI type materials, the mechanical properties of these semiconductors remain far from being well known and clearly understood.

$\mathrm{SnTe}$ is a promising both thermoelectric and topological insulator material. At temperatures from 20 to $800 \mathrm{~K}$ it has the regular (fcc) crystal structure of the rock-salt type [5]. Some physical properties of SnTe and values of its parameters (including the lattice parameter) strongly depends on the number of defects (SnTe is a non-stoichiometric crystal and the ratio of Sn:Te is always smaller than one).

The bulk crystals of (Sn,Mn)Te solid solutions aroused interest in the second half of the last century and most of their physical properties was determined during the

*corresponding author; e-mail: szusz@ifpan.edu.pl period 1970-1995. It had been demonstrated that these crystals exhibited a ferromagnetic phase and belonged to the diluted magnetic semiconductor (DMS) family (see, e.g., [6] and references therein). Over the last few years an alloying of SnTe with MnTe has been mostly considered as an efficient method of increasing the high SnTe thermoelectric performance [7-11].

Our interest in SnTe-based solid solutions is related to the recent discovery of topological crystalline insulator states at (001) and (111) surfaces of bulk SnTe crystals [3]. Lately, thin layers of SnTe-based materials with nonzero magnetization have been also grown by MBE and investigated at the Institute of Physics, PAS. The aim of the present studies is to investigate possible evolution of both microhardness and the Young modulus values in thin $(\mathrm{Sn}, \mathrm{Mn}) \mathrm{Te}$ layers with an increasing $\mathrm{Mn}$ content in the solid solution.

\section{Experimental details}

Three (Sn,Mn)Te solid solution thin layers with various chemical compositions (pure $\mathrm{SnTe}$ and samples for which about $4 \%$ and $8 \%$ of Sn was replaced by $\mathrm{Mn}$ ) were grown by $\mathrm{MBE}$ technique on (111)-oriented $\mathrm{BaF}_{2}$ substrates. The as-grown samples were characterized by X-ray diffraction (XRD), scanning electron microscopy (SEM), and energy dispersive X-ray spectrometry (EDX) measurements. All measurements have been performed at room temperature. The XRD was measured in a wide angular range (for $2 \theta$ values ranging from 20 to 140 degree) using laboratory X'Pert PANalytical diffractometer and $\mathrm{Cu} K_{\alpha_{1}}$ radiation. The lattice parameter value was determined for each sample by XRD. However, because of a huge concentration of native defects in SnTe its lattice parameter is strongly sample dependent and is not 
a fixed value for a given ( $\mathrm{Sn}, \mathrm{Mn}$ )Se crystal composition (see, e.g., [5]). Moreover, even for SnTe bulk crystal and thin layer the lattice parameter value is not the same as it has been demonstrated some time ago in [12]. Under the circumstances the chemical composition of $(\mathrm{Pb}, \mathrm{Mn}) \mathrm{Te}$ solid solution layers was determined by EDX measurements and the XRD data served only for the check of the crystal quality.

The surface morphology of all sample was measured by atomic force microscopy (AFM) technique. Finally, the data about microhardness and the Young modulus was determined by the nanoindentation method using an Ultra Nanohardness Tester CSM UNHT/AFM and the Berkovich intender tip. These values were obtained for the maximum load equal to $1 \mathrm{mN}$. Such load was applied during $15 \mathrm{~s}$, both the increase of the load during application and the removal of the load were performed in a linear manner with the same upload and download rate $0.033 \mathrm{mN} / \mathrm{s}$. The average values and standard deviations of the microhardness and the Young modulus were extracted from the determined load-displacement results.

\section{Results and discussion}

The analysis of diffraction patterns obtained for the investigated solid solutions confirmed the high crystal quality of all samples. As an example of such a diffraction pattern the data obtained by XRD for thin layer of $(\mathrm{Sn}, \mathrm{Mn})$ Te solid solution containing about $8 \%$ of $\mathrm{Mn}$ is shown in Fig. 1. All samples under studies exhibited the regular (fcc) crystal structure of the rock-salt type. The epitaxial mode of the crystal growth was demonstrated and the crystal orientation of all layers was the same as that of the substrate. A possible presence of other phases or precipitates was not detected.

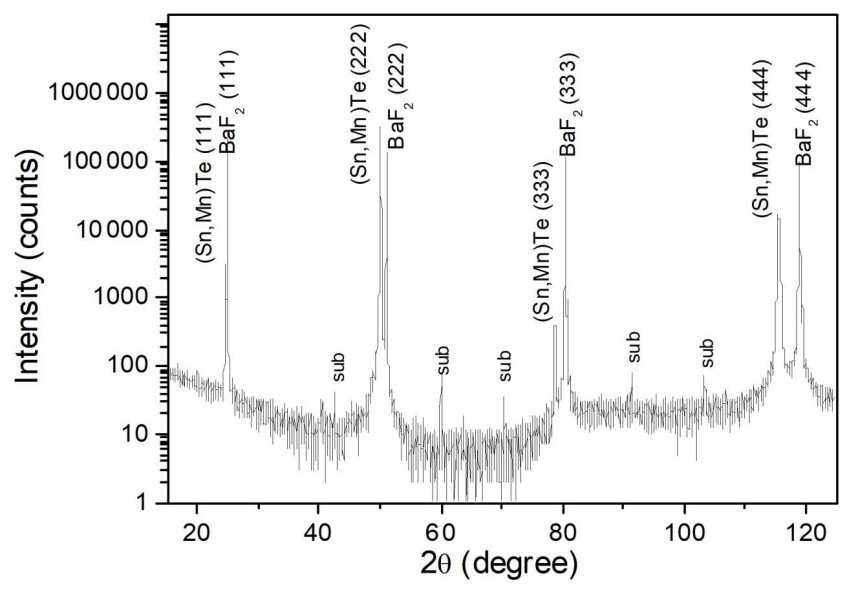

Fig. 1. The X-ray diffraction pattern for the thin layer of (Sn,Mn)Te solid solution containing about $8 \%$ of $\mathrm{Mn}$ measured by XRD.

Figure 2 presents the surface morphology for $\mathrm{SnTe}$ sample resulting from AFM measurements. According
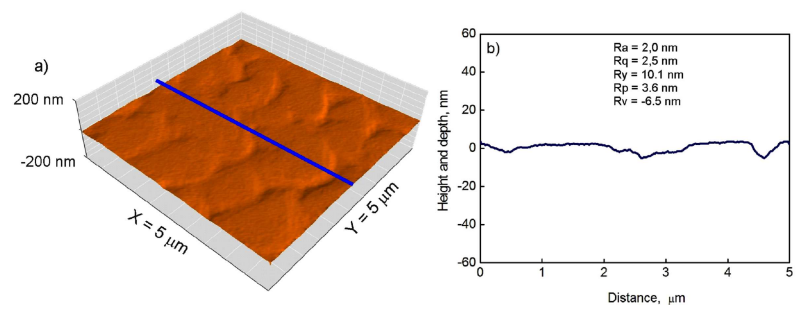

Fig. 2. (a) Surface topography of MBE-grown SnTe layer resulting from AFM measurements. (b) Surface profile along the line indicated in (a) and the relevant surface roughness parameters.

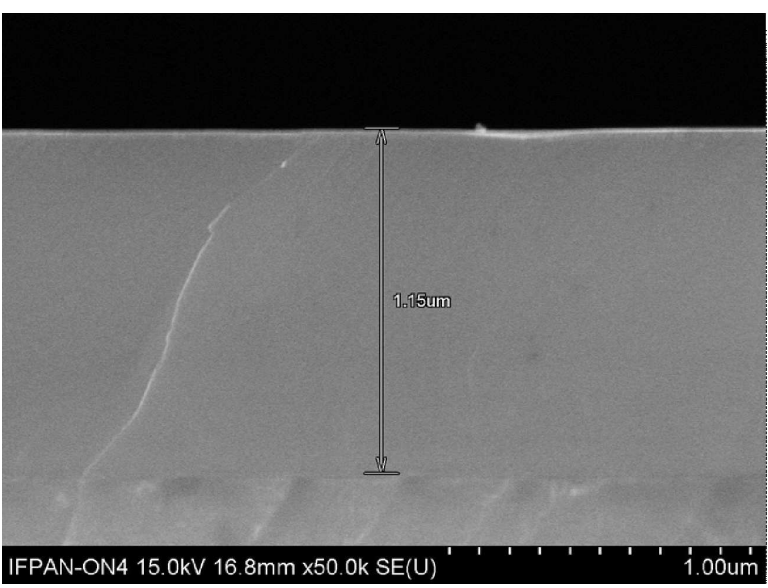

Fig. 3. The cross-section of $(\mathrm{Sn}, \mathrm{Mn})$ Te solid solution sample containing $4 \%$ of $\mathrm{Mn}$ (upper part) deposited onto $\mathrm{BaF}_{2}$ substrate (bottom part) and measured by SEM.

to the measured EDS spectra the solid solutions investigated in this paper contained 0,4 , or $8 \%$ of $\mathrm{Mn}$. The roughness for the SnTe sample was the smallest one and this value increased along with an increase of Mn content in the solid solution. The RMS value determined for the layer under interest, corresponding to the surface shown in Fig. $2 \mathrm{a}$ was equal to about $2.5 \mathrm{~nm}$, for the sample containing $8 \%$ of Mn the RMS value was about four times higher.

According to the results of SEM measurements the thickness of all thin layers was equal to about $1 \mu \mathrm{m}$ (Fig. 3).

The experimental curves presenting dependence of the nanoindentation depth on applied forces are shown in Fig. 4. As one can see a noticeable modification of the form of the curve with an increasing load is observed. In order to neglect possible influence of the substrate mechanical properties on the results corresponding to investigated layer the maximum nanoindentation depth should be considerably smaller than the total layer thickness. The values of the maximum indentation depth to the total layer thickness ratio which could be found in the literature varied from 4 to 10 . In our case this value is at least higher than 5 . 


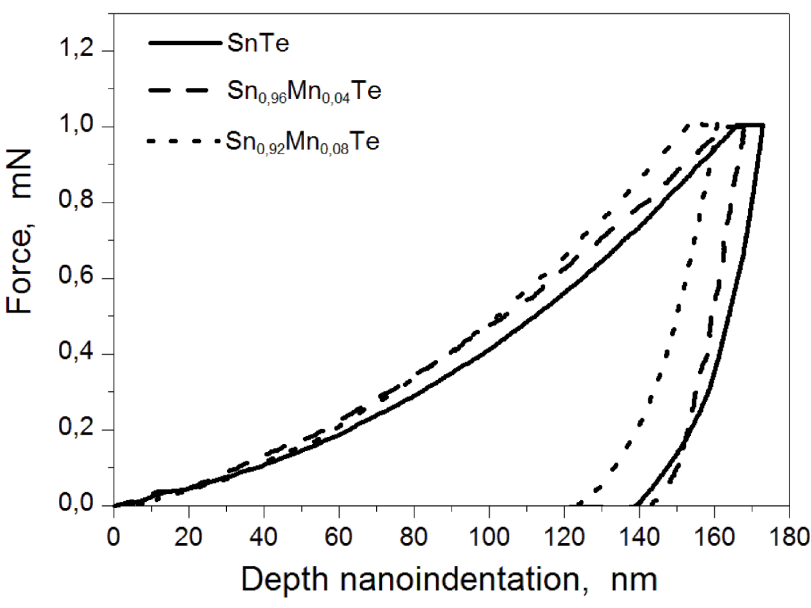

Fig. 4. The experimental curves load-nanoindentation depth determined for investigated $(\mathrm{Sn}, \mathrm{Mn}) \mathrm{Te}$ samples using nanoindentation.

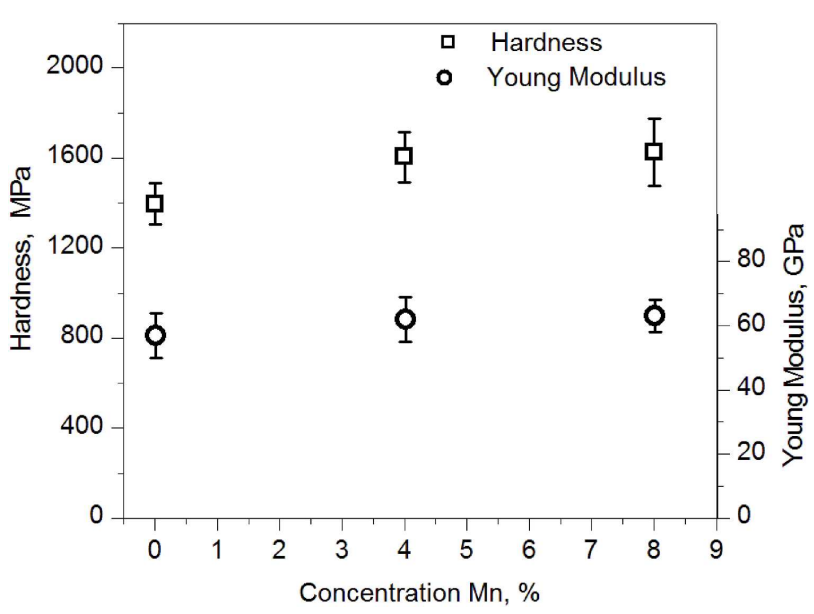

Fig. 5. The composition dependence of both microhardness and the Young modulus values determined for $(\mathrm{Sn}, \mathrm{Mn})$ Te investigated layers by the nanoindentation.

The interpretation of the results of the nanoindentation measurements is shown in Fig. 5. All numerical values given above are subjected to the experimental error of the order of at least $\pm 5 \%$. Within the experimental error the value of the the Young modulus does not depend on the chemical composition of the (Sn,Mn)Te solid solution and is equal to about $60 \mathrm{GPa}$. A slight increase of the layer microhardness with increasing $\mathrm{Mn}$ content (from about $1400 \mathrm{MPa}$ for SnTe layer to $1600 \mathrm{MPa}$ for the solid solution containing about $8 \%$ of $\mathrm{Mn}$ ) was found. It is difficult to compare values mentioned above with the relevant values corresponding to similar materials. The lattice parameter value in the ( $\mathrm{Sn}, \mathrm{Mn}) \mathrm{Te}$ solid solution decreases with an increase of Mn content but the mechanical properties of a hypothetical MnTe crystal with the rock-salt structure are not known. The MnTe crystallizes in the hexagonal structure of the NiAs type ([13] and references therein), the metastable, zinc blende phase of this compound only can also be obtained by MBE (see, e.g., [14]). The observed qualitative composition dependence of the microhardness in $(\mathrm{Sn}, \mathrm{Mn}) \mathrm{Te}$ thin layers is similar to that observed in $(\mathrm{Pb}, \mathrm{Sn}) \mathrm{Te}$ bulk crystal [15] but differs significantly from that recently observed for several PbTe-based solid solutions containing Cd [16, 17], Ge [18], or Ca [19]. For example using the same experimental setup the determined Young modulus was also a constant value in $(\mathrm{Pb}, \mathrm{Cd}) \mathrm{Te}$ solid solution but the microhardness increased by almost $50 \%$ in a similar composition range [17]. The microhardness of $(\mathrm{Pb}, \mathrm{Zn}) \mathrm{Se}$ and $(\mathrm{Pb}, \mathrm{Cd})$ Se solid solutions containing up to a few percent of $\mathrm{Zn}$ and $\mathrm{Cd}$, respectively, also significantly increases with an increasing dopant metal content [20]. In the case of $(\mathrm{Pb}, \mathrm{Ge}) \mathrm{Te}$ bulk crystals both the microhardness and the Young modulus values were significantly higher for the solid solution containing $10 \%$ of Ge than those for $\mathrm{PbTe}[18]$.

\section{Conclusions}

The mechanical properties of MBE-grown, thin (Sn,Mn)Te layers do not depend in a significant manner on the Mn content in the solid solution (at least up to the composition with $8 \%$ of $\mathrm{Mn}$ ). The Young modulus remains almost a constant value in the analyzed composition range, a slight increase of the microhardness with increasing Mn content is found. The observed composition dependence of selected mechanical properties mentioned above are in a qualitative agreement with those obtained for several IV-VI based solid solutions. The same time their numerical values differ significantly from the most of data corresponding to the PbTe-based solid solutions such as the $(\mathrm{Pb}, \mathrm{Cd}) \mathrm{Te}$ and $(\mathrm{Pb}, \mathrm{Ge}) \mathrm{Te}$ bulk crystals. The Young modulus determined at the identical conditions using the same experimental equipment was also a constant value in the $(\mathrm{Pb}, \mathrm{Cd}) \mathrm{Te}$ but the microhardness of this solid solution increased by almost $50 \%$ in a similar composition range.

\section{Acknowledgments}

This work was partly supported by National Science Centre (Poland) through grants No. UMO-2014/15/B/ST3/03833 and No. UMO2014/13/B/ST3/04393.

\section{References}

[1] E.S. Božin, C.D. Malliakas, P. Souvatzis, T. Proffen, N.A. Spaldin, M.G. Kanatzidis, S.J.L. Billinge, Science 330, 1660 (2010).

[2] O. Delaire, J. Ma, K. Marty, F. May, M. McGuire, M.-H. Du, D.J. Singh, A. Podlesnyak, G. Ehlers, M.D. Lumsden, B.C. Sales, Nat. Mater. 10, 614 (2011).

[3] Y. Tanaka, Z. Ren, T. Sato, K. Nakayama, S. Souma, T. Takahashi, K. Segawa, Nat. Phys. 8, 800 (2012). 
[4] P. Dziawa, B.J. Kowalski, K. Dybko, R. Buczko, A. Szczerbakow, M. Szot, E. Łusakowska, T. Balasubremanian, B.M. Wojek, M.H. Berntsen, O. Tjernberg, T. Story, Nat. Mater. 11, 1023 (2012).

[5] M. Sist, E.M. Jensen Hedegaard, S. Christensen, N. Bindzus, K.F. Fearch Fischer, H. Kasai, K. Sugimoto, B. Brummerstedt Iversen, Int. Union. Cryst. J. 3, 377 (2016).

[6] P.J.T. Eggenkamp, C.W.H.M. Vennix, T. Story, H.J.M. Swagten, C.H.W. Swüste, W.J.M.J. de Jonge, J. Appl. Phys. 75, 5728 (1994).

[7] J. He, X. Tan, J. Xu, G.-Q. H. Liu, H. Shao, Y. Fu, X. Wang, Z. Liu, J. Xu, H. Jiang, J. Jiang, J. Mater. Chem. A 3, 19974 (2015).

[8] W. Li, Z. Chen, S. Lin, Y. Chang, B. Ge, Y. Chen, Y. Pei, J. Materiom. 1, 307 (2015).

[9] G. Tan, F. Shi, S. Hao, H. Chi, T.P. Bailey, L. D. Zhao, C. Uher, C. Wolverton, V.P. Dravid, M.G. Kanatzidis, J. Am. Chem. Soc. 127, 11507 (2015).

[10] S.M. Li, J.Q. Li, L. Yang, F.S. Liu, W.Q. Ao, Y. Li, Mater. Des. 108, 51 (2016).

[11] S. Acharya, J. Pandey, A. Soni, Appl. Phys. Lett. 109, 133904 (2016).
[12] R. Diduszko, V. Domuchowski, A.J. Nadolny, J. Sadowski, Thin Solid Films 367, 168 (2000).

[13] W. Szuszkiewicz, E. Dynowska, B. Witkowska, B. Hennion, Phys. Rev. B 73, 104403 (2006).

[14] E. Janik, E. Dynowska, J. Bak, M. Leszczynski, W. Szuszkiewicz, T. Wojtowicz, G. Karczewski, A.K. Zakrzewski, J. Kossut, Thin Solid Films 267, 74 (1995).

[15] J.L. Cui, X. Qian, X.B. Zhao, J. Alloys Comp. 358, 228 (2003).

[16] A.J. Crocker, M. Wilson, J. Mater. Sci. 13, 833 (1978).

[17] R. Kuna, R. Minikayev, M. Trzyna, K. Gas, A. Bosak, A. Szczerbakow, S. Petit, J. Łażewski, W. Szuszkiewicz, Acta Phys. Pol. A 130, 1251 (2016).

[18] B. Li, P. Xie, S. Zhang, D. Liu, J. Mater. Sci. 46. 4000 (2011)

[19] A. Schmitz, J. de Boor, K. Mull, E. Müller, J. Mater. Sci. 51, 6933 (2016).

[20] R.D. Schmidt, E.D. Case, L.-D. Zhao, M.G. Kanatzidis, J. Mater. Sci. 50, 1770 (2015). 